\title{
« Tu es devenu toubab »
}

\section{Comprendre le retour temporaire au prisme des attentes de la famille restée au pays chez les migrants originaires d'Afrique subsaharienne}

\author{
Césarine Sambou
}

Émulations - Revue de sciences sociales 2020, n 34, "Transnationaliser le retour. Vers une révision du regard sur les migrations de retour contemporaines ».

\section{Article disponible à l'adresse suivante}

https://ojs.uclouvain.be/index.php/emulations/article/view/sambou

\section{Pour citer cet article}

Césarine Sambou, " "Tu es devenu toubab". Comprendre le retour temporaire au prisme des attentes de la famille restée au pays chez les migrants originaires d'Afrique subsaharienne », Émulations, n 34, Mise en ligne le 7 octobre 2020.

DOI : 10.14428/emulations.034.04

Distribution électronique : Université catholique de Louvain (Belgique) : ojs.uclouvain.be

(C) Cet article est mis à disposition selon les termes de la Licence Creative Commons Attribution, Pas d'Utilisation Commerciale 4.0 International. http://creativecommons.org/licenses/by-nc/4.0/

Éditeur : Émulations - Revue de sciences sociales / Presses universitaires de Louvain https://ojs.uclouvain.be/index.php/emulations

ISSN électronique : 1784-5734

$\frac{\text { PUL PRESSES }}{\text { UNIVERSITAIRES }}$




\title{
«Tu es devenu toubab »
}

\author{
Comprendre le retour temporaire au prisme des \\ attentes de la famille restée au pays chez les migrants \\ originaires d'Afrique subsaharienne
}

Césarine Sambou ${ }^{1}$

\begin{abstract}
[Résumé] La migration et le retour temporaire dans la société de départ sont des moments importants, tant pour le migrant que pour sa famille restée au pays. Les proches non migrants attendent beaucoup du migrant qui revient en termes de solidarité et de redistribution des richesses accumulées en Europe. À partir d'une enquête qualitative multisituée à Bordeaux auprès de migrants originaires d'Afrique subsaharienne et de leurs familles au Sénégal et au Burkina Faso, cette étude présente les enjeux du retour temporaire au prisme des attentes de la famille non migrante. Les résultats montrent comment les migrants originaires d'Afrique subsaharienne, qui rencontrent des difficultés à répondre aux attentes de la famille non migrante, sont étiquetés de « toubab», et les effets de cet étiquetage sur le plan social, familial et identitaire.
\end{abstract}

Mots clés : toubab, retour temporaire, attentes, migrants, Afrique subsaharienne, Bordeaux.

"You became a toubab": understanding the temporary return through the prism of the expectations of the family left behind among migrants from sub-Saharan Africa

[Abstract] Migration and temporary return to the sending society are important moments, both for the migrant and for his or her family back home. Non-migrant relatives expect a lot from the returning migrant in terms of solidarity and redistribution of accumulated wealth in Europe. Based on a qualitative multi-sited survey in Bordeaux among migrants from West Africa and their families in Senegal and Burkina Faso, this study presents the challenges of the temporary return to the prism of the expectations of the non-migrant family. The results show how these migrants who have difficulty meeting the expectations of the non-migrant family are labeled as "toubab" and the effects of this labelling on the social, family and identity dimensions.

Keywords: toubab, temporary return, migrant, expectations, Sub-Saharan Africa, Bordeaux.

\section{Introduction}

La littérature relative à la migration de retour en général et aux migrants africains en particulier s'est développée ces dernières années (Flahaux, Eggerickx, Schoumaker, 2017; Fouquet, 2007; Mondain, 2017; Riccio, 2002 ; 2006 ; Sayad, 1991). Elle montre que l'intention de revenir dans le pays d'origine est souvent présente chez les migrants au moment du départ (Diallo, 2015 ; Flahaux, 2013 ; Mazzella, 2014). Ces études ont montré que les migrants sont confrontés à de nombreux défis à leur retour au pays. Elles

\footnotetext{
${ }^{1}$ LAM (Les Afriques dans le monde), UMR 5115, Université de Bordeaux, France ; et CEPED (Centre population et développement), UMR 196, Université Paris Descartes-IRD, France.
} 
soulignent en outre l'importance de la situation économique, sociale, familiale dans la définition des intentions de retour dans leur pays d'origine et le rôle des ressources que les migrants ont pu acquérir en termes de capital économique, humain et social pour leur réinsertion. Le retour représente aussi un enjeu social important, en particulier en ce qui concerne la construction des identités et des statuts, la négociation des pratiques et des rapports de pouvoir au sein des communautés transnationales (Petit, 2007).

Cette littérature invite également à être vigilant sur l'usage de la notion de retour, qui s'avère difficile à conceptualiser en contexte de migration dans la mesure où elle peut correspondre à des réalités très différentes. La notion de retour peut renvoyer à un découpage de la circulation migratoire, à une séquence de déplacement que l'observateur extrait d'un contexte et d'une dynamique et non comme une fin en soi (Petit, 2007). Pour Bénédicte Michalon (2007), le terme désigne une migration au cours de laquelle l'ethnicité du migrant guide le processus migratoire. Ainsi, il n'y a pas un, mais des modèles de retour qui correspondent à des situations avec des implications parfois contradictoires, notamment les situations d'échec, de réussite, de circulation et d'adaptation auxquelles répond le retour. En effet, dans l'approche classique du retour, on distingue les retours forcés, qui sont inhérents aux politiques publiques migratoires, et les retours volontaires (Boyer, 20172), qui concernent souvent les personnes qui ont acquis des ressources humaines et économiques à l'étranger (Sinatti, 2015). Parmi les retours volontaires, il y a les retours circulaires où l'immigré rebondit vers d'autres lieux de fixation temporaire et les retours temporaires qui consistent en des allers et retours entre pays d'accueil et pays d'origine. Dans les deux derniers cas, le retour est replacé dans un système de circulation suivant une catégorisation calquée sur celle des migrations (Boyer, 2017 ; Petit, 2004).

Tout en étant très variées, les situations migratoires de retour entretiennent des relations étroites et complexes les unes avec les autres (Michalon, 2007). Le va-et-vient entre le pays d'installation et le pays de départ est donc une des modalités possibles du retour et constitue à l'heure actuelle une des formes les plus fréquentes de retour en raison de l'installation plus durable que prévu dans les pays d'accueil, et ce pour un ensemble complexe de raisons (économiques, familiales et politiques). Dans la pratique, le retour temporaire est davantage pensé et vécu comme une étape, un moment dans la trajectoire migratoire qui a pour fonction, entre autres, de compenser la non-concrétisation du retour définitif au pays d'origine souvent désigné comme un mythe (De Coulon, Wolff, 2006) et de s'adapter à l'évolution du contexte transnational (Mondain, 2017). Dans le cadre de cette étude, le retour temporaire s’inscrit dans cette approche et correspond à des visites individuelles ou en famille au pays d'origine pour passer les vacances, pour participer à des cérémonies religieuses ou traditionnelles et pour travailler (activités commerciales, entrepreneuriales et salariées). Ce sont des visites

${ }^{2}$ OCDE (2008), « Les migrations du retour : un nouveau regard », in OCDE, Perspectives des migrations internationales en 2008. En ligne, consulté le 1 er juillet 2020. URL : http://dx.doi.org/10.178./migr_outlook-2008-7-fr. 
temporaires sans intention de rester dans le pays d'origine pendant plus de trois mois et qui font partie intégrante du parcours migratoire.

À l'échelle individuelle, les motifs de l'émigration et du retour temporaire dépendent des facteurs socioculturels et économiques. La famille au sens large s'avère être un facteur déterminant de l'émigration et de l'intention du retour temporaire (Sambou, 2018). Une étude montre que la famille occupe une place centrale dans les comportements migratoires des jeunes (Maunaye, 2013). Dans la plupart des cas, elle a participé au projet migratoire en le finançant. En contrepartie, le migrant doit accumuler suffisamment de moyens afin d'assurer ce que la famille attend de lui, à savoir le bien-être de sa famille élargie. Ceci d'autant plus que dans beaucoup de sociétés africaines, fondées sur un système patriarcal se caractérisant par l'importance des liens de parenté et d'alliance, les migrants se trouvent insérés dans des réseaux de relations de réciprocité avec la famille élargie, incluant les membres de la parenté éloignée et d'alliance (Mondain, 2017). Dans ce contexte d'échanges et de réciprocité, certains migrants se trouvent dans l'obligation de soutenir et d'assister leur famille, notamment en lui transférant des fonds depuis le pays d'immigration et en répondant aux sollicitations lors des retours temporaires.

Par conséquent, retourner temporairement auprès des siens dans son pays d'origine requiert d'importantes ressources financières (Sambou, 2018) qui devraient permettre à l'émigré d'assumer les obligations et responsabilités qu'il a envers les membres de sa famille (Mondain, Diagne, Randall, 2012). Par ailleurs, des travaux s'intéressant au vaet-vient de migrants entre l'Europe et l'Afrique subsaharienne (Crenn, 2011 ; Mondain, 2017 ; Riccio, 2006) abondent dans le même sens. Nathalie Mondain (2017) montre comment les femmes migrantes qui circulent entre l'Italie et le Sénégal sont elles aussi fortement sollicitées par leur famille proche ou éloignée pour un soutien régulier ou des contributions de type cadeaux, ou lors de l'organisation de cérémonies qui jalonnent la vie des Sénégalais. Son article relève également l'importance capitale de la redistribution de leurs richesses pour le maintien et le développement des réseaux de parenté élargie. Nonobstant, les attentes de la famille non migrante peuvent être « démesurées » et " pressantes » (Diome, 2005; Sambou, 2018).

Si toutefois les ressources sont insuffisantes et que le migrant rencontre des difficultés à répondre aux attentes de la famille restée au pays, il peut se voir accuser de «toubabisation ». Emprunté à Philippe Lavigne-Delville (1991) et à Thomas Fouquet (2008), le terme "toubabisation " vient du verbatim toubab, couramment utilisé dans beaucoup de pays ouest-africains pour désigner le « Blanc ${ }^{3} »$. Dans le cadre de cette étude, la « toubabisation » est une construction socioculturelle et identitaire pour qualifier le migrant qui a des manières de faire et de vivre à l'occidentale, et qui ne procède pas à la redistribution de la richesse accumulée en Europe. Comme le souligne Chantal Crenn (2011), la famille restée au pays n'attend pas que les migrants soient « absolument traditionnels » lors du retour temporaire. Elle demande qu'ils correspondent dans leurs

\footnotetext{
${ }^{3}$ Au Burkina Faso, on utilise aussi le terme Nasara.
} 
comportements aux critères de richesse que l'on attribue à l'Occident et qu'ils soient " généreux » en partageant cette richesse avec elle. Ces attentes peuvent mettre le migrant dans une position d'entre-deux, où il doit osciller entre " être un peu traditionnel » et « être riche comme en Occident » lors du retour temporaire. Cette ambivalence met en exergue la complexité identitaire du migrant partagé entre deux cultures, entre deux réalités sociales et économiques (Santelli, 2010). Par conséquent, il peut avoir le sentiment de n'appartenir entièrement à aucune société, ni dans le pays d'origine ni dans le pays d'accueil (Sayad, 1991). En outre, la « toubabisation » vue comme telle est dans ce contexte utilisée par la famille comme une manœuvre stratégique (Fouquet, 2008) pour réaffirmer la solidarité familiale et comme un moyen de pression sur celui qui revient afin qu'il réponde à ses exigences. C'est dans cette perspective que cet article ambitionne d'analyser l'expérience du retour temporaire de Bordeaux des migrants originaires des pays d'Afrique subsaharienne, et de montrer comment les attentes de la famille non migrante peuvent créer de véritables bouleversements sur les plans social, familial, statutaire et identitaire.

Une première partie traitera de la méthodologie utilisée pour étudier le retour temporaire. Dans la deuxième partie, nous décrirons les attentes de la famille non migrante et les perceptions de ces attentes par les migrants eux-mêmes. Enfin, nous analyserons à partir de l'expérience de la «toubabisation » les contournements prévus et adoptés par les migrants pour minimiser ce risque et pour se repositionner au niveau de la hiérarchie familiale et sociale.

\section{Les conditions d'enquête : une ethnographie multisituée pour étudier le retour temporaire}

Cet article s'inscrit dans un programme de recherche doctorale sur la prévention du risque de paludisme lors des séjours temporaires en Afrique subsaharienne. ${ }^{4}$ Au départ, les voyageurs migrants originaires d'Afrique subsaharienne à Bordeaux étaient la principale population cible. La mise à plat des premières données a fait émerger une nouvelle question : celle du retour temporaire. Au regard de cette nouvelle problématique et pour mieux comprendre et analyser les enjeux du retour temporaire, il nous a semblé utile d'inclure les familles non migrantes restées au pays d'origine. Notre propos s'articule autour de onze mois d'enquête à Bordeaux, au Sénégal et au Burkina Faso entre novembre 2014 et septembre 2015. Les résultats s'appuient sur soixante-huit entretiens en face à face et des discussions informelles avec des migrants originaires d'Afrique subsaharienne résidant à Bordeaux, ainsi qu'avec des personnes de leur entourage dans leur pays d'origine.

L'enquête de terrain a été réalisée en deux étapes. La première phase de terrain était consacrée aux migrants originaires d'Afrique subsaharienne et a eu lieu au service

\footnotetext{
${ }^{4}$ Cette thèse avait pour principal objectif d'analyser la question du recours à la prévention du risque palustre auprès des migrants originaires des pays d'Afrique subsaharienne de Bordeaux.
} 
santé voyages ${ }^{5}$ du Centre hospitalier universitaire (CHU) de Bordeaux ainsi qu'à leur domicile dans la communauté urbaine de Bordeaux. Quarante-sept migrants ont été interrogés, dont vingt-trois au service santé voyages et vingt-quatre à domicile. Les immigrés résidant à Bordeaux et dans son agglomération ayant acquis ou non la nationalité française, et nés à l'étranger dans les pays d'Afrique de l'Ouest, ont été considérés comme migrants originaires d'Afrique subsaharienne. Je me suis intéressée seulement à ceux qui étaient en situation régulière, car, pour pouvoir circuler entre l'Europe et l'Afrique légalement, il faut un titre de séjour valide. Les enquêtés étaient âgés de 25 à 59 ans et résidaient en France depuis au moins deux ans. La durée maximale de résidence était de quarante ans. La plupart des migrants ayant participé à cette enquête étaient des hommes (trente et un). Les seize femmes rencontrées dans le cadre de cette étude ont été interrogées au service santé voyages en compagnie de leurs enfants venus pour les démarches de prévention et de vaccination internationales. Parmi les quarante-sept migrants ayant participé à la recherche, huit étaient des étudiants au moment de l'enquête et trente-neuf des travailleurs immigrés (dont six cadres supérieurs, cinq autoentrepreneurs et vingt-huit salariés). La quasi-totalité des migrants qui avaient le statut de travailleur étaient mariés : vingt-deux vivaient avec leurs époux·se·s en France et six avaient leurs époux·se-s dans leur pays d'origine. Les entretiens ont porté, entre autres, sur les préparatifs, l'organisation du voyage, les différentes préoccupations et les enjeux du retour temporaire.

Quant à la deuxième phase de terrain, elle s'est intéressée aux personnes de l'entourage (parents, frères, sœurs, époux·se-s) de dix migrants interrogés au service santé voyages $^{6}$. Il faut noter que les migrants rencontrés au service santé voyage étaient originaires de plusieurs pays d'Afrique subsaharienne, notamment la Côte d'Ivoire, la Guinée, le Niger, le Tchad, le Nigéria, le Gabon, la République centrafricaine, le Burkina Faso, le Sénégal et la République démocratique du Congo. Tous préparaient un voyage, et nous en connaissions les dates prévues. Pour des raisons pratiques et économiques, nous avons choisi le Sénégal et le Burkina Faso pour réaliser la deuxième phase de l'enquête. Dans cette deuxième phase, nous avons réalisé vingt et un entretiens. Les participants étaient issus des dix familles de migrants ayant accepté que leur entourage participe à l'étude. Dans six de ces familles, nous avons interrogé de deux à trois membres, parfois dans des localités différentes. Au Sénégal, douze personnes de l'entourage ont participé à l'étude, dont cinq hommes et sept femmes. Les entretiens ont eu lieu pour moitié lors de la visite des migrants, mais en leur absence. Au Burkina Faso, l'enquête de terrain a eu lieu quelques mois après le retour des migrants et a concerné

\footnotetext{
${ }^{5}$ Le service santé voyages est l'unité fonctionnelle qui dépend du service interne des maladies tropicales du CHU de Bordeaux (Hôpital Saint-André). Il est le principal centre de consultations, de conseils et de vaccinations internationales pour les voyageurs de Bordeaux et des alentours.

${ }^{6}$ Pour réaliser la deuxième phase de terrain qui concerne l'entourage, le consentement du migrant était un préalable au recrutement de l'entourage : il a été demandé aux migrants interrogés à santé voyages s'ils acceptaient que leur entourage resté dans le pays d'origine soit enquêté à son tour. Après leur accord, leurs contacts et les contacts de leur entourage nous ont été fournis pour demander dans un second temps le consentement de l'entourage avant d'organiser les entretiens.
} 
neuf personnes, dont quatre hommes et cinq femmes. Le guide d'entretien a porté sur leurs perceptions de la migration et de l'Europe, les attentes de l'entourage vis-à-vis du migrant qui revient, et sur les relations qu'ils entretiennent avec le migrant. Les personnes de l'entourage avaient des âges compris entre 19 et 74 ans et près de la moitié étaient des femmes. Un dispositif d'enquête bien défini a été mis en place : tous les entretiens ont été enregistrés avec l'accord du participant et retranscrits intégralement ${ }^{7}$ avant de faire l'objet d'une analyse de contenu.

\section{Les attentes de la famille non migrante}

Le retour temporaire est fondamental pour beaucoup de migrants, mais également pour leurs familles restées au pays. La rencontre entre le migrant et sa famille lors du retour temporaire demeure aussi un moment de confrontation entre imaginaire et réalité de la migration. En effet, la population restée sur place continue de considérer l'Europe comme un eldorado (Riccio, 2005). La migration, du point de vue de beaucoup de familles africaines, est associée à la réussite et est perçue comme une des voies les plus rapides de réussite sociale, culturelle et économique (Sambou, 2018). L'association de la migration à la réussite prend source dans les regards croisés entre les migrants et les familles non migrantes, en confrontant l'ici (pays d'immigration) et l'ailleurs (pays d'origine), la réalité et le désir, l'abstrait et le concret. Ces regards croisés lors du retour temporaire s'articulent autour de « dialogues imaginaires » (Fouquet, $2008: 244$ ), dont les logiques doivent être recherchées dans un jeu d'énonciation de l'altérité et de l'identité. Le passage du migrant est pour la société d'origine une aubaine de comparaison entre les normes sociales, les cultures, les modes de vie de la société d'origine (in group) et de la société autre (out group) (Koumba, 2014).

Cet imaginaire migratoire, qui est nourri en partie par des images diffusées dans les médias télévisés, est ancré dans un processus migratoire transnationalisé où une culture de la migration se maintient et se construit à travers l'observation de l'expérience des migrants. Les transferts d'argent qui creusent les écarts entre les bénéficiaires et les autres (Mondain, Diagne, Randall, 2012) et la procuration des biens de consommation de luxe à caractère ostentatoire sont aussi des éléments qui contribuent au maintien de cet imaginaire. Ainsi, la population restée au pays et qui n'a pas l'expérience migratoire peut voir ceux qui reviennent de l'Europe comme des «portefeuilles à prendre », nous disait Jules, un jeune cadre sénégalais de $32 \mathrm{ans}^{8}$. Et la famille non migrante va attendre beaucoup du migrant en termes de solidarité et de générosité, qui se caractérise par la redistribution de cette supposée richesse accumulée en Europe. La crise économique qui a touché de plein fouet l'Afrique renforce les attentes et les exigences vis-à-vis des solidarités communautaires.

\footnotetext{
${ }_{7}^{7}$ Nous avons également tenu un journal où étaient recueillies des notes de terrain. Les noms et les prénoms des interlocuteurs ainsi que les indications trop précises pouvant les identifier ont été modifiés pour préserver leur anonymat.

8 Jules, Sénégalais, 32 ans, marié, cadre, Bordeaux, en France depuis 8 ans, entretien réalisé le 19 mars 2015.
} 
Cette solidarité, généralement perçue comme une assurance sociale plus que jamais nécessaire en temps de crise (Leimdorfer, Marie, 2003) et comme un moyen de maintenir les rapports, se manifeste souvent avant le retour temporaire par des transferts réguliers de fonds du migrant à sa famille. Toutefois, elle ne doit pas être interrompue lorsque la personne qui réalise les remises est auprès des bénéficiaires. En effet, lors du retour temporaire, en plus du billet d'avion et des dépenses liées aux démarches de santé (vaccins, chimioprophylaxie antipaludique'), les migrants originaires d'Afrique subsaharienne sont confrontés à d'autres dépenses, en l'occurrence celles pour les cadeaux et celles pour assurer les dépenses quotidiennes, ainsi que les imprévus, alors qu'ils avaient déjà envoyé de l'argent à cet effet. Ainsi, dès leur arrivée, ils ont la charge de toutes les dépenses quotidiennes de leur famille, l'obligation de réparer tout ce qui ne fonctionne pas et d'acheter tout ce qui manque dans la maison, comme l'illustre ces deux extraits d'entretiens :

Aller au pays c'est bon, tu seras en famille et tout, mais en même temps en regardant bien ça ne nous arrange pas, car une fois là-bas, tu vas dépenser tout ce que tu avais comme économies. Je m'occupe des dépenses quotidiennes de la maison. Et quand tu rentres ici tu recommences à zéro. Tu ne peux pas réaliser quelque chose dans ces conditions ${ }^{10}$.

Dans ma propre famille, quand j'arrive là-bas il y a des choses qui ne marchent pas à la maison et je suis obligé de les réparer. Il y a des choses qui manquent, je suis obligé d'acheter et quand ils voient ça ils se disent : « Lui, il a beaucoup d'argent ${ }^{11}$ ! »

La situation générale, telle qu'elle vient d'être décrite à grands traits, permet de comprendre le rôle que les migrants sont appelés à jouer quand ils retournent périodiquement dans leur pays d'origine. Le migrant qui revient, en plus de gérer les dépenses quotidiennes et imprévues, doit offrir des cadeaux à son entourage proche (membres de la famille, amis, voisins, etc.).

C'est obligé d'acheter des cadeaux. Tu connais l'Afrique, non ? Les gens n'attendent que ça. Dès que tu arrives, la première des choses, c'est : qu'est-ce que tu nous as apporté ? Je ne peux pas aller chez moi après plusieurs années d'absence sans rien ramener. Ça fait trois ans que je n'ai pas vu ma famille, on discute souvent sur Skype, mais ce n'est pas pareil. [...] Tu sais que les gens dès que tu es en France, ils pensent que tu as une bonne situation. Donc il faut leur acheter des cadeaux. La famille, surtout les jeunes frères et sœurs, et les amis n'attendent que ça. Pour eux, c'est l'occasion de goûter un peu à la migration [...]. Pour mes parents, les cadeaux ne les intéressent pas trop. Eux, quand tu ar-

\footnotetext{
${ }_{9}^{9}$ Conformément aux recommandations sanitaires pour les voyageurs à destination des pays à risque palustre, les voyageurs migrants originaires des pays d'Afrique subsaharienne doivent se procurer une chimioprophylaxie antipaludique et certains vaccins en vue d'éviter la survenue de problèmes de santé.

10 Ousmane, Burkinabé, 34 ans, célibataire, agent de sécurité, Talence, en France depuis 9 ans, entretien réalisé le 6 novembre 2014.

${ }^{11}$ Djibril, Franco-Sénégalais, 59 ans, divorcé, retraité par anticipation, Bordeaux, en France depuis 40 ans, entretien réalisé le 4 décembre 2014.
} 
rives, tu leur donnes une somme d'argent et ils sont plus contents. Franchement je ne peux y aller sans rien amener ${ }^{12}$.

Le cadeau pour nous, c'est important. C'est une marque d'affection pour nous, sa famille. Ça permet de montrer qu'il nous aime et que la famille est importante et ça aide à ne pas oublier qui il est pour nous ${ }^{13}$.

Les cadeaux sont incontournables. Ils créent et actualisent ou font évoluer les relations sociales. Le don de cadeaux et les mécanismes qui le régissent sont révélateurs des relations qu'entretiennent les migrants avec leur famille restée au pays. Les raisons de ce don sont liées entre elles et de manière paradoxale. Ainsi, le migrant qui donne n'est susceptible de satisfaire son propre intérêt que par le détour de la satisfaction de l'intérêt de son entourage (Andrys, 2000). En effet, si certains objets relèvent bien du système de don, d'autres ont un statut plus ambigu (Chevalier, 2009). Pour cet auteur, amener dans ses bagages des objets synonymes de modernité, de progrès estampillés et de « mode de vie à la française » constitue autant de modalités complexes de dynamiques qui permettent au migrant d'instaurer un système relativement cohérent qui garantit à la fois l'identité symbolique et sociale du migrant et la cohésion de la famille, voire de la communauté dans son ensemble. La peur de trahir, de décevoir les siens est forte et pousse les migrants à réaliser de multiples achats pour montrer qu'ils font toujours partie de la famille. Le fait d'offrir un présent venu de la France n'est pas uniquement une manière de manifester son amour, mais une manière de ne pas se faire blâmer, et de (re)dorer son image. Par son geste, le migrant se positionne, au moins partiellement, à l'abri de la critique. Il bénéficie en retour d'une position avantageuse. Les destinataires se souviendront à chaque fois du don lorsqu'on parle du donateur. La valeur de chaque cadeau doit être estimée à l'aune de la distance de provenance et du lien avec le bénéficiaire (Diome, 2005).

En explorant les perceptions des migrants ayant participé à l'étude sur leur retour temporaire au pays d'origine et les logiques autour desquelles s'organise le retour temporaire, on constate une tendance à légitimer les attentes de la famille non migrante. Les migrants interrogés considèrent qu'il est tout à fait normal et légitime que la famille restée au pays compte sur eux. Il est de leur devoir d'aider leurs proches dans le besoin. Le sens du devoir est transmis depuis la socialisation primaire de l'enfant (Wright, 2010). Les parents inculquent à l'enfant le sens du respect des obligations envers autrui en général, et plus particulièrement envers la famille au sens large. Comme la famille étendue demeure ainsi leur lieu de socialisation communautaire, les individus y sont sommés, quelle que soit la force de leur individualité, de mettre celle-ci au service de leur communauté d'origine. Dans le contexte migratoire, ce devoir de solidarité est considéré comme une « tradition », entretenue en partie par les premières vagues migratoires (Fall, 2011). Dans les pays en voie de développement, la migration est toujours valorisée comme une ressource où le migrant apparaît à la fois comme pourvoyeur ma-

${ }^{12}$ Bintou, Sénégalaise, 41 ans, mariée, aide à domicile, Bordeaux, en France depuis 7 ans, entretien réalisé le 21 novembre 2014.

${ }^{13}$ Yandé, Sénégalaise, 47 ans, mariée, enseignante, Dakar, entretien réalisé le 6 mai 2015. 
tériel de sa famille et agent de développement local potentiel (Tall, 2008). La perception positive de la migration a fait émerger la construction d'un migrant qui se « sacrifie » pour sa famille, ce qui peut se révéler contre-productif par la suite pour l'épanouissement de celui-ci (Phongi, Mabika Mabika, 2015). En outre, la famille non migrante inscrit sa demande dans une morale fortement normative (Koumba, 2014), basée sur le devoir de partage et de redistribution considéré comme symétriquement opposé au procès de « toubabisation ». Cette morale permet de créer des normes sociales de redistribution variables selon les personnes et selon le degré d'implication.

Toutefois, sans toujours vouloir l'imposer sous une forme autoritaire, ceux restés au pays exercent néanmoins une pression diffuse et considérable sur les migrants. Plusieurs auteurs (Dia, 2007 ; Dimé, 2013) ont fait ce constat et montrent que les migrants sont l'objet de pressions familiales ; les sommes demandées sont en constante augmentation d'une année à l'autre. Ces auteurs ajoutent que cette augmentation est rarement à l'initiative individuelle du migrant. En effet, ils sont pris en otage par un système qui fait d'eux des « esclaves qui produisent pour entretenir les gens qui vivent mieux psychologiquement qu'eux », nous disait Djibril ${ }^{14}$. Pour ce dernier, les migrants sont devenus au fil du temps des instruments d'une tradition qui est falsifiée et modernisée pour devenir profiteuse et capitaliste.

La pression est beaucoup plus importante pour ceux qui rencontrent des difficultés à satisfaire les sollicitations de la famille non migrante ou pour les migrants dont les ressources s'épuisent au cours du séjour. Dans ce contexte, ils sont souvent l'objet de jugements négatifs construits sur l'adoption de pratiques et de modes de pensée de « toubab », ici critiqué et stigmatisé pour son manque de générosité. Les migrants qui sont étiquetés de « toubab» subissent un regard stigmatisant et dévalorisant, une attitude qui leur renvoie l'image d'étrangers dans leur pays d'origine.

\section{«Toubab », de quoi parle-t-on?}

«Toubab » est un terme utilisé dans beaucoup de pays ouest-africains, particulièrement au Sénégal, pour désigner l'« Européen », principalement le « Blanc ». Mais ce terme acquiert un sens plus large et devient synonyme des façons d'agir et de penser qui seraient propres à l'Occident, c'est-à-dire manquer de solidarité, en dépit de sa richesse matérielle, ne pas manifester suffisamment d'hospitalité et, au bout du compte, manquer de dignité. En d'autres termes, le migrant qualifié de « toubab » devient un symbole négatif, combinant tous « les défauts » condamnés dans les sociétés africaines (Riccio, 2002). Le migrant qui ne se comporte pas comme un «big man » (Laurent, 2012), c'est-à-dire qui redistribue les ressources en fonction des pressions exercées par la famille qui l'entoure lors du retour temporaire dans son pays d'origine, est étiqueté de « toubab», car, pour la famille non migrante, «tukki badola ñibisi buur » (« on voyage pauvre on revient riche », en

14 Djibril, Franco-Sénégalais, 59 ans, divorcé, retraité par anticipation, Bordeaux, en France depuis 40 ans, entretien réalisé le 4 décembre 2014. 
wolof). Ainsi, la personne qui revient d"Europe ne peut pas manquer de moyens financiers comme elle le prétend. Donc, le migrant a un comportement nouveau acquis en Europe et se fait passer pour un individualiste occidental, un égoïste, comme l'illustrent les propos de Lathat, un Sénégalais de 28 ans qui travaille dans la restauration et réside en France depuis six ans lors de notre rencontre le 26 février 2015:

L'expérience est toujours là quand on vient ici. Que ce soit votre famille ou une autre personne, ils croient que tu as des millions dès que tu reviens de la France. Ils croient qu'on a des millions parce qu'on est venu au mois de juin et même pas un an on est revenu [...]. Tout ce que je peux vous affirmer, ma propre famille c'est-à-dire ma mère, mes frères, les enfants de mes frères et leurs épouses, s'intéressent à l'argent. Si tu leur dis que tu n'as pas d'argent, ils ne te croiront pas. C'est eux qui vont commencer à dire aux gens de l'extérieur que tu es devenu mauvais. Tu es avare, tu es égoïste [...]. Tu es devenu une mauvaise personne, que la France t'a complètement changé. Je suis devenu toubab. Et tout cela complique la relation avec la famille au sens large.

Ainsi, le migrant qualifié de « toubab » est celui qui agit autrement que ce que la société d'appartenance attend de lui en termes de solidarité, de redistribution, mais aussi en termes de comportement. L'accusation de " toubab » est socialement moins acceptée par les migrants dans leur société d'origine. En outre, cette accusation est vécue comme une offense au regard de leur dévotion envers la famille et des travaux endurés en Europe, ne serait-ce que pour leur rendre visite. Ce qualificatif remet en cause leur identité. Le migrant a perdu son " africanité ", les valeurs communautaires en vigueur pour devenir un Occidental. Or, les migrants sont attachés à leur identité même si l'attachement identitaire compense un défaut qu'il aggrave par ailleurs (Rey, 2007). Le procès de " toubabisation » fonctionne comme un dispositif de contrôle social auquel est soumis le migrant. Le retour temporaire confronte aussi les migrants à un problème de réadaptation sociale et culturelle, ce qui provoque des réactions négatives de la part de la famille non migrante. La difficulté pour les migrants retournant dans leur pays de naissance de s'exprimer spontanément en langue locale sans y ajouter le français contribue aussi au procès de «toubabisation ». Des expressions banales telles que "vous n'êtes pas en France ici », " c'est un toubab maintenant », expriment l'idée de l'éloignement culturel. Cela amène le migrant à se considérer comme une figure en mouvement plus jamais chez lui, et en quête de place. Cette expérience éprouvée lors du retour permet de mesurer le cheminement accompli et rend le migrant natif étranger en son pays (De Gourcy, 2007). Il est dans son pays d'origine sans y être, et sans y retrouver ses repères familiaux. Le migrant fait l'objet de relations à l'« étranger » dans sa propre société d'appartenance. Ainsi, revenir au pays équivaut pour certains à partir, car une fois sur place, on devient l'autre pour ceux que nous continuons à appeler les nôtres. Cette dynamique est particulièrement activée quand l'individu s'engage dans un processus de change- 
ment personnel qui comporte l'immersion temporaire dans l'autre société. Ce qui constitue un enjeu supplémentaire pour celui qui retourne pour quelque temps dans son pays d'origine.

\section{Contournements adoptés pour éviter le risque de " toubabisation "}

Comme il ne répond pas aux attentes parfois pressantes et démesurées de la famille, le migrant peut aussi perdre son statut : il doit souvent renoncer à son rang de "Grand ", une expression courante utilisée dans beaucoup de pays d'Afrique de l'Ouest pour exprimer le rapport d'aînesse civile, de séniorité sociale et, dans le contexte migratoire, le respect et la réussite sociale. Crenn (2011) montre comment des retraités et des préretraités ont perdu leur statut social « d'aînés » et de " vieux entourés et respectés » et se sont vus ostracisés pendant leurs séjours respectifs et réguliers à Dakar à cause de leur manque de générosité. Elle précise qu'en réalité, la place du migrant fortuné n'est pas suffisante pour assurer et maintenir le statut d'aîné si le migrant ne fait pas don de cette richesse lors des cérémonies religieuses. Il est donc nécessaire de manifester cette richesse accumulée en Europe.

Le non-respect des obligations et des « devoirs » aboutit, dans certaines communautés, à la marginalisation du migrant ou à son exclusion du cercle décisionnel (Mondain, Diagne, Randall, 2012). D’autres travaux (De Latour, 2003 ; Jamoulle, 2013 ; Timéra, 2001) abondent dans ce sens et montrent que les migrants qui n'ont pas réussi leur projet migratoire, et ne rentrent donc pas en « héros », sont considérés comme des «morts sociaux ». Par conséquent, ils ne détiennent aucun pouvoir de décision qui correspond à leur statut, au profit de l'aîné « économique », bouleversant ainsi les rapports de pouvoir et les rôles familiaux et implicitement la relation entre le migrant et sa famille. Dans cette situation, des conflits intergénérationnels peuvent survenir au sein d'une même famille, qui à leur tour peuvent ébranler les équilibres familiaux et traditionnels. L'intérêt de la famille restée au pays pour l'argent et la perte de l'autorité pour le migrant peuvent contribuer à rendre inconfortables les relations entre les migrants et leur famille d'origine.

Réussir son retour temporaire tout en évitant le risque de « toubabisation » constitue un défi pour beaucoup de migrants qui ont participé à cette recherche. Pour ce faire, il est important, en plus de bien se préparer, de réaliser des séjours courts et de résider en dehors de la maison familiale.

\subsection{Se préparer économiquement}

La satisfaction des attentes de la famille constitue une préoccupation majeure pour la plupart des migrants ayant participé à cette recherche. Les décisions de retourner temporairement dans le pays pour quelques moments sont fonction du statut juridique et en partie de la situation sur le marché du travail dans le pays d'accueil. Autrement 
dit, la situation financière dans le pays d'accueil peut amener le migrant à revenir plus vite que prévu quand le travail en migration le lui permet. Des travaux (Flahaux, 2013; Sinatti, 2011) ont suggéré que même si l'intention de retourner dans le pays d'origine est souvent présente chez les migrants, cette décision de retourner nécessite d'être bien préparée afin de minimiser les risques. D’autres auteurs ont montré combien les attentes que les familles placent à l'endroit des migrants les obligent à économiser pour se donner les moyens d'assumer certaines responsabilités sociales (Mondain, Diagne, Randall, 2012). Ainsi, pour bien se préparer, la moitié des migrants interrogés ont économisé pendant plusieurs mois, d'autres pendant plusieurs années, pour supporter toutes les charges qui les attendent dans leur pays d'origine. La fréquence du retour temporaire dépendrait de la capacité pour le migrant à économiser. Nous avons rencontré des migrants qui retournaient régulièrement (tous les deux ans) dans leur pays d'origine alors que d'autres repartaient occasionnellement, à intervalles irréguliers. D’autres encore retournaient pour la première fois dans leur pays d'origine après avoir passé plus de huit ans en France, parce qu'ils avaient jugé qu'ils n'avaient pas atteint leurs objectifs financiers.

On ne peut pas se lever comme ça et partir à la hâte. II faut bien toujours mettre de l'argent de côté pour se préparer financièrement. C'est pourquoi on ne va pas souvent parce que c'est devenu de plus en plus dur ici. Les Blancs ne gagnent pas plus que nous. La seule différence c'est que nous, notre salaire c'est pour plusieurs personnes. Si ce que je gagne était pour moi seul, je peux mettre de côté facilement de l'argent pour aller chez moi. [...] Si on n'économise pas assez rapidement d'argent, on ne peut pas y aller et si on voyage, à qui on va donner et laisser les autres ? On risque de faire des jaloux. On doit être prêt sur le volet financier. Je connais des Africains qui ont leurs papiers et qui travaillent, qui ont fait plus de huit ans en France avant de retourner dans leur pays. Au début je les critiquais, mais maintenant je les comprends. Ce n'est pas facile du tout de partir en Afrique sans moyens financiers ${ }^{15}$. »

Lorsque l'argent économisé est jugé insuffisant par les migrants pour pouvoir supporter les charges, il leur arrive de contracter des prêts à la banque, comme c'est le cas de Saliou, un autoentrepreneur franco-sénégalais de 42 ans. Il est en France depuis seize ans. Depuis le décès de son père en 2010, Saliou repartait tous les deux ans au Sénégal pour rendre visite à sa mère, qui s'est retrouvée seule dans la maison familiale. Avant de partir, il économisait, comme à l'accoutumée dit-il, pendant plusieurs mois. Ces économies lui ont permis à chaque voyage de répondre aux attentes de sa mère ainsi que de ses frères et sœurs. En février 2016, il décide de retourner temporairement avec son épouse (franco-sénégalaise) et leurs deux enfants. Un an avant le voyage, Saliou et son épouse ont essayé de mettre de côté de l'argent pendant plusieurs mois. Mais, par expérience, Saliou a jugé que l'argent qu'ils avaient pu accumuler n'était pas suffisant pour satisfaire aux besoins de sa propre famille ainsi qu'à ceux de sa belle-fa-

${ }^{15}$ Samba, Burkinabé, 43 ans, marié, agent de sécurité, Pessac, en France depuis 11 ans, entretien réalisé le 6 janvier 2015. 
mille. Ils ont ainsi contracté un prêt de 6000 euros à la banque afin de pouvoir supporter les charges. Grâce à cet emprunt, ils ont réussi à tenir, même si la peur que la somme s'épuise avant la date prévue du retour était omniprésente. La place importante qu'occupe dans les discours le désir de satisfaire les attentes de la famille et, implicitement, le souhait d'être toujours considérés comme l'un des leurs, pousse nombre de migrants à espacer leurs retours pour avoir le temps de mieux se préparer ${ }^{16}$.

Dans une logique de réduction du risque d'épuisement des économies pendant le séjour, certains migrants réalisent des séjours courts. Car, dès qu'ils n'ont plus d'argent, toutes les personnes qu'ils ont pu aider disparaissent. Eux qui étaient le centre d'intérêt de l'entourage et qui étaient célébrés pour leur solidarité et les efforts qu'ils avaient déployés pour le bien-être de leurs familles (Riccio, 2005) sont désormais délaissés avec le risque d'être étiquetés de «toubab».

Les vacances en Afrique, il n'y a pas mieux ! Mais, il faut avoir beaucoup d'argent. Quand je dis beaucoup, c'est vraiment beaucoup, car il y a pas mal de dépenses à faire sur place et il faut tenir jusqu'au retour sinon quand tu n'auras plus rien, les gens te tournent le dos. Ils se moquent de toi en disant des trucs du genre: «On savait qu'il n'a pas grand-chose et qu'il n'allait pas aller plus loin ! » Pour éviter tout ça, je ne reste pas trop longtemps sinon les gens vont te dépouiller, mais aussi tu risques de rentrer avec plein de surnoms [...] comme tu es maudit ${ }^{17}$.

En réalisant des séjours courts, le migrant a plus de chance de suivre la cadence dépensière des proches. Cela permet également au migrant de sauver son image et sa réputation. La durée du séjour semble être un facteur d'évaluation du risque de « toubabisation ». Les séjours longs de plusieurs mois augmenteraient les dépenses dans la durée et exposent plus le migrant au risque de «toubabisation » et à des problèmes financiers. Des travaux ont prouvé l'association de séjours de longue durée à un risque accru de survenue de problèmes, notamment sociaux et sanitaires (Palvi et al., 2014 ; Toovey et al., 2007).

\subsection{Habitat individuel}

Nombreux sont les migrants qui cherchent aussi leur bien-être personnel. Même s'ils n'avouent pas explicitement leur individualisme, les propos de Seydou le sous-entenden $t^{18}$. Il est enseignant à l'université au Sénégal et sa famille vit à Bordeaux. Pour l'équilibre familial, il fait des va-et-vient entre le Sénégal et la France. Pour lui, la famille est importante, mais, à un certain âge, il faut aussi penser à soi, construire sa vie. Ainsi, il opte pour l'unité résidentielle en louant un appartement au lieu d'habiter avec sa famille. Cette stratégie de distanciation vis-à-vis de la famille permet d'après lui d'échapper aux valeurs communautaires dominantes qui ne favorisent pas l'épanouis-

\footnotetext{
${ }^{16}$ Saliou, Franco-Sénégalais, 42 ans, marié, autoentrepreneur, Talence, en France depuis 16 ans, entretien réalisé le 11 février 2015.

17 Angélique, Ivoirienne, 32 ans, célibataire, cuisinière, Talence, en France depuis 13 ans, entretien réalisé le 22 décembre 2014.

18 Seydou, Sénégalais, 31 ans, marié, enseignant, Cenon, en France depuis 9 ans, entretien réalisé le 27 mars 2015.
} 
sement personnel, qui menacent l'autonomisation et qui font obstacle à l'individualisation (Marie, 1997). Certains migrants sont fortement mus par le besoin d'échapper aux pressions et à l'ordre social afin d'évoluer dans un nouvel ordre social dans lequel ils seront - parce qu'éloignés de chez eux - plus en mesure de résister aux pressions sociales et familiales tout en faisant de leur mieux pour répondre à leurs obligations vis-à-vis de leurs parents. Les enquêtés semblent ainsi répondre à une certaine obéissance mesurée, donnant l'apparence d'adhérer aux normes de redistribution tout en tentant d'y échapper. Cette obéissance concerne des migrants qui, conscients du poids que représente la vie en famille, essaient de limiter la charge de la redistribution en décidant de couper le cordon.

Les propos d'Ismaël, Burkinabé de 31 ans, fournissent un exemple édifiant en montrant qu'habiter ou séjourner loin de la famille dans son pays d'origine est un choix de maturation et de responsabilisation, bien que difficile à admettre par la famille ${ }^{19}$. Pour Ismaël, la migration résidentielle peut permettre de rendre la situation financière du migrant moins observable ou de garder un certain flou autour des revenus. Marie Boltz-Laemmel et Paola Villar (2013) ont apporté un éclairage similaire dans leur article portant sur les liens des migrants internes et internationaux à leur ménage d'origine au Sénégal. Pour ces auteures, pour échapper aux normes de redistribution, la plupart des migrants internationaux se lient les mains en investissant dans des biens « non partageables » ou en optant pour « une migration résidentielle, car les revenus réels des contributeurs hors ménage d'origine sont moins observables du fait de la distance géographique » (Boltz-Laemmel, Villar, 2013 : 116). La distanciation par l'unité résidentielle traduit également un désir d'autonomie des migrants. Une étude menée auprès d'une lignée forgeronne dans la vallée du fleuve Sénégal (Diallo, 2015) analyse l'autonomie dans une logique de compétition et de négociation. Cette autonomie surgit dans tout le processus de migration ; elle prend forme dans la mise en place du projet migratoire et se manifeste après le retour.

Ce choix avant tout stratégique, et porté à la fois par la volonté d'autonomisation et le désir d'initiative personnelle, soulève la problématique de la bonne distance (Maunaye, 2013) et son effet sur les liens familiaux. Cet acte de retrait qui isole le migrant du reste de sa famille d'origine est perçu comme une des solutions pour parvenir à une autonomie financière loin des pressions familiales dans un contexte économique local bloqué. Toutefois, il est important de ne pas couper les liens avec la famille. Il faut continuer à maintenir les relations familiales, notamment en communiquant régulièrement avec elle et en passant des journées en famille tout en maintenant une certaine distance.

\section{Conclusion}

Cet article montre les enjeux économiques, sociaux, familiaux et identitaires rattachés au retour temporaire. Les migrants sont heureux de retourner temporairement

19 Ismaël, Burkinabé, 31 ans, célibataire, comptable, Bruges, en France depuis 6 ans, entretien réalisé le 14 janvier 2015. 
auprès des leurs, mais reconnaissent néanmoins le fardeau économique et social que le retour temporaire représente. L'analyse de ces enjeux montre que la migration et le retour temporaire dans la société de départ sont des moments importants, tant pour le migrant que pour sa famille restée au pays. Même si le migrant se sent harcelé et noyé par les demandes d'aides, il admet néanmoins la part de gratification qu'il y a à se sentir ainsi « quelqu'un d'important » lorsqu'il parvient à mettre de côté ses aspirations personnelles au profit de celles de la famille. Quant à la famille, elle exprime ses attentes et en retire une forme d'amertume lorsque le migrant ne les satisfait pas. Cette ambivalence du rapport entre le migrant et la famille non migrante crée généralement une conflictualité (Fouquet, 2008). En effet, lors du séjour temporaire, les migrants qui osent agir autrement que d'habitude parce qu'ils n'ont pas les moyens nécessaires pour le faire peuvent perdre ce qu'ils avaient acquis (identité, statut, considération, pouvoir de décision) (Lenoble-Bart, Damone, 2015). Ils peuvent être assimilés à des « figures intermédiaires d'identité et de différence » (Bhabha, 2007 : 29), que l'on peut admirer ou critiquer en fonction des circonstances. Dans cette perspective, le migrant qui retourne temporairement dans son pays d'origine devient un point focal de toute l'ambivalence du rapport entretenu stratégiquement avec l'extranéité, et doit ainsi faire preuve de « tactiques » pour le maintien d'une ethnicité (comme rapport social) qui lui permette d'être lui-même dans son pays d'origine.

Cet article pointe du doigt la sensibilité du retour temporaire et l'inconfortable situation des migrants retournant chez eux depuis la France, écrasés par les attentes démesurées de ceux qui sont restés au pays. Les difficultés croissantes du migrant à faire face aux contraintes quotidiennes peuvent aboutir à une crise de confiance et d'autorité. Ainsi, même s'ils ont peur d'épuiser leurs économies accumulées en France pendant leur séjour, ils s'efforcent de se dépouiller du peu qu'ils ont pour minimiser le risque de "toubabisation », tant pour le respect des obligations morales que pour le maintien de leur statut, montrant ainsi l'ambivalence du retour temporaire.

Cette recherche illustre les déconvenues du retour temporaire au pays d'origine. Les prises de distance tout comme les rapprochements vis-à-vis de la famille s'élaborent de manière ambivalente et montrent que, pour des raisons économiques, beaucoup de migrants ne sont plus libres de retourner temporairement auprès des leurs pour tirer un peu le bénéfice individuel de leur expérience migratoire. La complexité des situations décrites et vécues par les immigrés nous encourage à replacer le phénomène du retour temporaire dans un processus plus large de transformations sociales induites par la globalisation des sociétés contemporaines. Selon cette perspective, nous rejoignons Mondain (2017) pour considérer le retour temporaire comme partie intégrante de ce processus et non un fait isolé ; il devient alors nécessaire de chercher à savoir s'il existe une continuité et/ou des variabilités de perception du retour dans le temps et au-delà de l'ethnicité et d'analyser la place réelle du migrant dans les relations et les dynamiques transnationales. 


\section{Bibliographie}

AndRys C. (2000), " Les cadeaux : règles et itinéraires des pratiques de don », in I. Garabuau-Moussaoui, D. Desjeux (dir.), Objet banal, objet social. Les objets quotidiens comme révélateurs des relations sociales, Paris, L’Harmattan, p. 155-172.

Bhabha H.K. (2007), Les lieux de la culture. Une théorie postcoloniale, Paris, Payot.

Boltz-Laemmel M., Villar P. (2013), « Les liens des migrants internes et internationaux à leur ménage d'origine : portraits croisés de familles étendues sénégalaises ", Autrepart, vol. 4-5, n 67-68, p. 103-119.

Boyer F. (2017), « Les migrants nigériens expulsés d'Arabie Saoudite. Une trajectoire dominée par l'incertitude », Espace populations sociétés, $n^{\circ} 1$. En ligne, mis en ligne le $1^{\text {er }}$ juin 2017, consulté le 23 octobre 2019. URL : http://journals.openedition.org/ eps/7088. DOI : $10.4000 /$ eps.7088.

Chevalier D. (2009), « Ici et là-bas : entre territorialités et l'entre deux et morcellements identitaires », Espaces, Populations Sociétés, nº 2, p. 293-304.

CRenN C. (2011), «"Vieillir" dans deux pays : une situation alimentaire "combinée " pour les migrants retraités "sénégalais" de Bordeaux », Gérontologie et société, vol. 34, $n^{\circ} 139$, p. 99-115.

De coulon A., WolfF F.-C. (2006), The location of Immigrants at Retirement: Stay/Return or "Va-et-Vient", Bonn, Institute of the Study Labour (IZA)(2224).

De Goucy C. (2007), « Le retour au prisme de ses détours ou comment réintroduire de la proximité dans l'éloignement », Revue européenne des migrations internationales, vol. 23, n² 2, p. 159-171.

De Latour E. (2003), « Héros du retour », Critique internationale, n 19, p. 171-189.

DiA H. (2007), "Confiance et conflits d'intérêt : le difficile investissement au pays des migrants de la vallée du fleuve Sénégal », Revue Européenne des Migrations Internationales, vol. 23, $\mathrm{n}^{\circ}$ 3, p. 29-49.

Diallo S. (2015), « Parcours familiaux, situations migratoires et stratégies d'autonomie: le cas d'une lignée forgeronne de la vallée du fleuve Sénégal (XX ${ }^{\mathrm{e}} \mathrm{XXI}{ }^{\mathrm{e}}$ siècles) », in P. Kamdem (dir.), Migrations et développement dans les Afriques de l'Ouest et du Centre, Paris, L’Harmattan, p. 71-91.

Dime M. (2013), « Comment investir si on doit faire face à autant de problèmes ? Enjeux et contraintes à la promotion de l'investissement chez les émigrés de retour au Sénégal », in G. Azoulay, V. Ancey, D. Dormoy, C. Crenn, A. Mangu, A. Thomashausen (dir.), Mobilités et Migrations : figures et enjeux contemporains. Pluralités des regards et des disciplines, Paris, L’Harmattan («Presses universitaires de Sceaux »), p. 135-149.

Diome F. (2005), Le ventre de l'Atlantique, Paris, Anne Carrière.

FLAHAUX M.-L. (2013), Retourner au Sénégal et en RD Congo. Choix et contraintes au cœur des trajectoires de vie des migrants, Louvain-la-Neuve, Presses universitaires de Louvain. 
Flahaux M.-L., Eggerickx T., Schoumaker B. (2017), « Les migrations du retour en Afrique », Espace populations sociétés, $\mathrm{n}^{\circ}$ 1. En ligne. URL : http://journals.openedition.org/ eps/7081. DOI : https://doi.org/10.4000/eps.7081.

FoUQUET T. (2007), « Imaginaires migratoires et expériences multiples de l’altérité : une dialectique actuelle du proche et du lointain », Autrepart, vol. 1, n 41, p. 83-97.

Fouquet T. (2008), « Migrations et globalisation dakaroise », in M. C. Diop, Le Sénégal des migrations. Mobilités, Identités et Sociétés, Paris, CREPOS/Karthala/ONU Habitat, p. 305-319.

Jamoulle P. (2013), Par-delà les silences. Non-dits et ruptures dans les parcours d’immigration, Paris, La Découverte.

Koumba T. (2014), Expérience migratoire et changement de stéréotypes sociaux, Paris, Publibook (« Sciences humaines et sociales »).

LAURENT P.J. (2012), " Engager l'anthropologie du développement à prendre en considération le malaise postcolonial ", Bulletin de l'A.P.A.D, $\mathrm{n}^{\circ}$ 34-36. En ligne, consulté le 5 mai 2020. URL : http://apad.revue.org/4108.

Lavigne-Delville P. (1991), " Le sanglot de l'homme noir », Cahiers d'études africaines, $\mathrm{n}^{\circ}$ 121-122, p. 231-240.

LEIMDORFER F., MARIE A. (dir.) (2003), L’Afrique des citadins. Sociétés civiles en chantier, Dakar/ Abidjan, Kharthala (« Homme et Société : Sciences économiques et politiques »).

Lenoble-Bart A., Damone E. (2015), «Introduction », in S. Pomel, Du risque en Afrique. Terrains et Perspectives, Paris/Pessac, Karthala/MSHA, p. 8-12.

MARIE A. (dir.) (1997), L'Afrique des individus. Itinéraires citadins dans l'Afrique contemporaine (Abidjan, Bamako, Dakar, Niamey), Paris, Karthala.

MAUnAyE E. (2013), « La migration des jeunes : quelles mobilités ? Quels ancrages ? La place des liens familiaux et des relations intergénérationnelles ", Enfances Familles Générations, $n^{\circ} 19$. En ligne, mis en ligne le 31 décembre 2013, consulté le $1^{\text {er }}$ juillet 2020. URL : http://journals.openedition.org/efg/1639.

Mazzella S. (2014), Sociologie des migrations, Paris, Presses universitaires de France.

Michalon B. (2007), " Les retours en migration : une notion polysémique, des formes migrations multiples ", in V. PETIT, Les migrations internationales de retour et pays d'origine, Paris, CEPED, p. 27-44.

Mondain N. (2017), " Migration, transnationalisme et reproduction sociale », Espace populations sociétés. En ligne, mis en ligne le $1^{\text {er }}$ juin 2017, consulté le 3 avril 2018. URL : http://journals.openedition.org/eps/7083. DOI : 10.4000/eps.7083.

Mondain N., Diagne A., Randall S. (2012), « Migration et responsabilités intergénérationnelles : implications pour la transition à l'âge adulte des jeunes migrants sénégalais ", in M. GOMEz-PEREZ, M.N. LEBLANC, L'Afrique des générations entre tensions et négociations, Paris, Karthala, p. 259-297. 
Palvi A., Smeti P., Spilioti A., Silvestros C., Katerelos P., Maltezou H. (2014), « Vaccinations and malaria prophylaxis for long-term travelers travelling from Greece: A prospective, questionnaire-based analysis », Travel Medicine and Infectious Disease, $\mathrm{n}^{\circ} 12$, p. 764-770.

Petrt V. (2007), « Présentation “Il n'est de richesse que d'hommes en mouvement...” », in V. PetrT, Les migrations internationales de retour et pays d'origine, Paris, CEPED, p. 9-26.

Phongi A., Mabika Mabika C. (2015), «Transferts d'argent et investissements économiques: part de la famille et expérience des congolais installés en Europe », in P. KAMDEM (dir.), Migrations et développement dans les Afriques de l'Ouest et du Centre, Paris, L’Harmattan, p. 155-190.

REY O. (2007), « Le symptôme identitaire », Études, vol. 6-7, p. 52-62.

Riccio B. (2002), "Toubab and Vu Cumprà: Italian perceptions of Senegalese», in R. D. GRillo, J. Pratt (dir.), The Politics of Recognizing Difference: Multicultaralism italian-Style, Farnham, Ashgate Publishing (" Research in Migrantion and Ethnic Relation »).

Riccio B. (2005), « Talking about migration. Some ethnographic notes on the ambivalent representation of migrants in contemporary Senegal ", Journal of african studies, p. 100-118.

Riccio B. (2006), « "Transmigrants”, mais pas "nomades” », Cahiers d'études africaines, $\mathrm{n}^{\circ}$ 181. En ligne, mis en ligne le $1^{\text {er }}$ janvier 2008, consulté le 24 mars 2018. URL : http://journals.openedition.org/etudesafricaines/15142.

SAmBou C. (2018), Le paludisme du retour. Une anthropologie du risque palustre auprès des migrants originaires des pays d'Afrique subsaharienne de Bordeaux, thèse de doctorat, Bordeaux, Université de Bordeaux.

SANTElli E. (2010), « Entre ici et là-bas : les parcours d'entrepreneurs transnationaux. Investissement économique en Algérie des descendants de l'immigration algérienne de France », Sociologie, n 3, vol. 1, p. 393-411.

SAYAD A. (1991), L’immigration ou le paradoxe de l'altérité, Bruxelles, De Boeck.

SinatTi G. (2011), « Mobile Transmigrants or Unsettled Returnees? Myth of Return and Permanent Resettlement among Senegalese Migrants », Population, Space and Place, $\mathrm{n}^{\circ}$ 17, p. 153-166. DOI : 10.1002/psp.608.

SiNATTI G. (2015), « Return migration as a win-win-win scenario? Visions of return among Senegalese migrants, the state of origin and receiving countries », Ethnic and Racial Studies, $n^{\circ} 38$, vol. 2, p. 275-291. DOI : 10.1080/01419870.2013.868016.

TALL S.M. (2008), « La migration internationale sénégalaise : des recrutements de maind'œuvre aux pirogues », in M.-C. Diop (dir.), Le Sénégal des migrations. Mobilités, identités et sociétés, Paris, Karthala, p. 37-68.

Timera M. (2001), "Les migrations des jeunes sahéliens : affirmation de soi et émancipation », Autrepart, vol. 2, n 18, p. 37-49. 
Toovey S., Moerman F., Van Gompel A. (2007), "Special infectious disease risks of expatriates and long-term travellers in tropical countries. Part I : Malaria », J. Travel Med., n 14. DOI : 10.1111/j.1708-8305.2006.00091.x.

WRIGHT R. (2010), The moral animal: why we are, the way we are: The new science of evolutionary psychology, New York, Knopf Doubleday Publishing Group. 